\title{
Reduced Representation Bisulfite Sequencing Determination of Distinctive DNA Hypermethylated Genes in the Progression to Colon Cancer in African Americans
}

\author{
Hassan Ashktorab, ${ }^{1}$ Afnan Shakoori, ${ }^{2,3}$ Shatha Zarnogi, ${ }^{2}$ \\ Xueguang Sun, ${ }^{4}$ Sudhir Varma, ${ }^{5}$ Edward Lee, ${ }^{6}$ Babak Shokrani, ${ }^{6}$ Adeyinka O. Laiyemo, \\ Kareem Washington, ${ }^{2}$ and Hassan Brim ${ }^{6}$ \\ ${ }^{1}$ Department of Medicine and Cancer Center, Howard University, Washington, DC, USA \\ ${ }^{2}$ Department of Genetics, Howard University, Washington, DC, USA \\ ${ }^{3}$ Umm AL-Qura University, Makkah, Saudi Arabia \\ ${ }^{4}$ DNA Sequencing and Genotyping Core, Cincinnati, OH 45229, USA \\ ${ }^{5}$ Hithru Analytics, Laurel, MD, USA \\ ${ }^{6}$ Department of Pathology, Howard University, Washington, DC, USA
}

Correspondence should be addressed to Hassan Ashktorab; hashktorab@howard.edu and Hassan Brim; hbrim@howard.edu

Received 13 November 2015; Accepted 7 April 2016

Academic Editor: Ralf-Dieter Hofheinz

Copyright (C) 2016 Hassan Ashktorab et al. This is an open access article distributed under the Creative Commons Attribution License, which permits unrestricted use, distribution, and reproduction in any medium, provided the original work is properly cited.

\begin{abstract}
Background and Aims. Many studies have focused on the determination of methylated targets in colorectal cancer. However, few analyzed the progressive methylation in the sequence from normal to adenoma and ultimately to malignant tumors. This is of utmost importance especially in populations such as African Americans who generally display aggressive tumors at diagnosis and for whom markers of early neoplasia are needed. We aimed to determine methylated targets in the path to colon cancer in African American patients using Reduced Representation Bisulfite Sequencing (RRBS). Methods. Genomic DNA was isolated from fresh frozen tissues of patients with different colon lesions: normal, a tubular adenoma, a tubulovillous adenoma, and five cancers. RRBS was performed on these DNA samples to identify hypermethylation. Alignment, mapping, and confirmed CpG methylation analyses were performed. Preferential hypermethylated pathways were determined using Ingenuity Pathway Analysis (IPA). Results. We identified hypermethylated CpG sites in the following genes: L3MBTL1, NKX6-2, PREX1, TRAF7, PRDM14, and NEFM with the number of CpG sites being 14,17,10,16,6, and 6, respectively, after pairwise analysis of normal versus adenoma, adenoma versus cancer, and normal versus cancer. IPA mapped the above-mentioned hypermethylated genes to the Wnt/ $\beta$-catenin, PI3k/AKT, VEGF, and JAK/STAT3 signaling pathways. Conclusion. This work provides insight into novel differential CpGs hypermethylation sites in colorectal carcinogenesis. Functional analysis of the novel gene targets is needed to confirm their roles in their associated carcinogenic pathways.
\end{abstract}

\section{Background}

Colorectal cancer (CRC) is the most common gastrointestinal cancer in the United States [1]. It is a major cause of cancerrelated mortality [2]. CRC occurs through different molecular mechanisms and pathways, leading to different clinical and pathological outcomes. CRC occurs more frequently in African Americans (AAs) compared to any other racial group in the United States $[1,3,4]$. The reasons for this disparity include environmental factors as well as genetic specific predispositions [1]. It is now widely recognized that, in addition to genetic mutations, epigenetic mechanisms especially aberrant DNA methylation are involved in virtually every step of CRC development and progression.

DNA methylation is one of the most important epigenetic events that is thought to occur during the early stages of such oncogenic transformation [5]. CpG islands, the target for methylation, consist of short stretches of $\mathrm{CpG}$ rich regions 
that are often associated with promoter regions of genes [6]. In neoplastic cells, there is a modest global depletion of cytosine methylation but considerable acquisition of aberrant methylation within certain promoters' associated $\mathrm{CpG}$ islands [7]. This aberrant promoter methylation generally leads to epigenetic silencing of gene expression [7].

The aberrant methylation of $\mathrm{CpG}$ islands within gene promoters and/or first exonic/intronic regions is a recognized epigenetic event that leads to transcriptional silencing of corresponding tumor suppressor genes in CRC and other cancers. Regardless of the biological consequences of methylation-induced silencing of tumor suppressor genes, this epigenetic alteration constitutes a molecular signature that can serve as promising biomarkers for early detection [3, 8]. Many genes are silenced by aberrant methylation in CRC (APC, p16INK4a, and TIMP3); such genes have the potential to become useful biomarkers for early detection of colorectal neoplasia [3, 8-10]. Early detection of colonic lesions is the most effective approach to reduce CRC incidence and mortality [11]. Therefore, molecular studies aimed at identifying CRC-specific methylation markers may provide useful insight for a better understanding of CRC progression $[12,13]$.

Most colorectal cancers arise from adenomatous polyps [14]. The progression to colorectal cancer is marked by specific ordered events that drive the "Adenoma-Carcinoma Sequence." Methylation in an average of 14 genes is required for the formation of malignant tumors and fewer changes only suffice for benign tumorigenesis [15]. Even though genetic alterations occur according to a preferred sequence, the total accumulation of changes rather than their order is responsible for the tumors progression and behavior.

DNA methylation can target genes involved at different stages and in different cellular pathways. Promoter methylation of CHFR (checkpoint with fork-head and ring-finger domains) was found to be associated with survival and was considered to be an independent predictor for tumor recurrence. IGFBP3 (insulin-like growth factor binding protein 3) and CD109 DNA methylation were associated with worse survival for stage II CRC. Tumor suppressor genes (TSG) including SOCS3, TFAP2E, and CDH4 have also been reported as DNA hypermethylation targets of which the gene expression was silenced in CRC tumors [16-19].

In this study, we investigated distinctive DNA methylation targets in normal, adenoma, and cancer specimens from African Americans using Reduced Representation Bisulfite Sequencing to detect novel DNA methylation targets in the path to cancer.

\section{Materials and Methods}

2.1. Patients and Tissue Samples. The samples were obtained from African American (AA) patients at Howard University Hospital (HUH) who underwent colonoscopy and/or surgery for the removal of colonic lesions (Table 1). All samples were analyzed by an expert gastrointestinal pathologist of the HUH Department of Pathology and kept frozen until used for DNA extraction. The samples consisted of a blood sample, a normal colon tissue, a tubular adenoma, a tubulovillous adenoma, and 5 cancers. These cancers were included to
TABLE 1: Demographical characteristics of the analyzed samples.

\begin{tabular}{lccc}
\hline Sample type & Gender & Age & Location \\
\hline Normal blood & Male & 60 & NA \\
Normal colon tissue & Male & 60 & Right \\
Tubular adenoma & Male & 59 & Left \\
Tubulovillous adenoma & Female & 76 & Right \\
Tumor & Male & 51 & Left \\
Tumor & Female & 69 & Right \\
Tumor & Female & 68 & Right \\
Tumor & Male & 55 & Left \\
Tumor & Female & 71 & Left \\
\hline
\end{tabular}

NA: not applicable.

select genes that are consistently methylated at the cancer stage. Clinical, demographic, and pathological data were collected for all specimens (Table 1). Informed consent was obtained from all participants. This research was approved by Howard University Institutional Review Board (IRB 06MED-39).

2.2. DNA Extraction. Genomic DNA was extracted from fresh frozen tissues. The samples were homogenized in lysis buffer consisting of $100 \mathrm{mM}$ Tris- $\mathrm{HCl}$ (pH 8.5), 5 mM EDTA, $0.2 \%$ SDS, and $200 \mathrm{mM} \mathrm{NaCl}$. Proteinase $\mathrm{K}$ was freshly added at a final concentration of $300 \mu \mathrm{g} / \mathrm{mL}$. Samples were incubated overnight at $55^{\circ} \mathrm{C}$ to ensure that genomic DNA is completely dissociated from any DNA binding proteins. After digestion, genomic DNA was extracted using a QIAGEN's genomic DNA extraction kit (Germantown, CA), according to the manufacturer's instructions (AllPrep QIAGEN kit). DNA, quality, and quantity were assessed using a NanoDrop spectrophotometer and $0.8 \%$ agarose gel electrophoresis.

2.3. EpiQuest Library Construction. EpiQuest libraries were prepared according to Zymo Research Protocol (Irvin, CA). 200-500 ng of genomic DNA were digested with NEB 60 units of TaqI and 30 units of MspI (Ipswich, MA, USA) sequentially. Size-selected TaqI-MspI fragments (40$120 \mathrm{bp}$ and $120-350 \mathrm{bp}$ ) were filled-in and $3^{\top}$-terminal-A extended, extracted with Zymo Research (ZR) DNA Clean and Concentrator ${ }^{\mathrm{TM}}-5 \mathrm{kit}$ (Irvin, CA). Ligation to preannealed adapters containing $5^{\prime}$-methyl-cytosine was performed using Illumina's DNA preparation kit and protocol (San Diego, CA). Purified adaptor ligated fragments were bisulfite-treated using the EZDNA Methylation-Direct ${ }^{\mathrm{TM}}$ Kit (Irvin, CA). Preparative-scale PCR was performed. DNA Clean and Concentrator-purified PCR products were subjected to a final size selection on a $4 \%$ NuSieve $3: 1$ agarose gel. SYBRgreen-stained gel slices containing adaptor-ligated fragments of $130-210 \mathrm{bp}$ or $210-460 \mathrm{bp}$ in size were excised. Library material was recovered from the gel (Zymoclean ${ }^{\mathrm{TM}}$ Gel DNA Recovery Kit, Irvin, CA, USA) and sequenced on an Illumina HiSeq Genome Analyzer (San Diego, CA).

2.4. EpiQuest Sequence Alignments and Data Analysis. Sequence reads from bisulfite-treated EpiQuest libraries were 
TABLE 2: Identified hypermethylated genes in colon neoplasia progression.

\begin{tabular}{lcccccc}
\hline Genes & $\begin{array}{c}\text { Spearman } \\
\text { correlation }\end{array}$ & $\begin{array}{c}\text { Adjusted } P \text { value } \\
\text { (false detection rate) }\end{array}$ & Normal & $\begin{array}{c}\text { Tubular } \\
\text { adenoma }\end{array}$ & $\begin{array}{c}\text { Tubulovillous } \\
\text { adenoma }\end{array}$ & $\begin{array}{c}\text { Tumor } \\
\text { L3MBTL1 }\end{array}$ \\
NKX6-2 & 0.22 & 2.93 & 0.0057 & 0.002353 & 0.00784 & 0.09 \\
PREX1 & 0.08 & 0.000158 & 0.0144 & 0.00375 & 0.0000 \\
TRAF7 & 0.16 & 0.03 & 0.0057 & 0.0000 & 0.121 & 0.12 \\
PRDM14 & 0.20 & 0.000244 & 0.0000 & 0.0000 & 0.0000 & 0.06 \\
NEFM & 0.24 & 0.01 & 0.0000 & 0.0000 & 0.0000 & 0.27 \\
\hline
\end{tabular}

identified using standard Illumina base-calling software and then were analyzed using a Zymo Research proprietary analysis pipeline according to the manufacturer's recommendations (Zymo Research, CA, USA). Residual cytosines (Cs) in each read were first converted to thymines (Ts), with each such conversion noted for subsequent analysis. A reference sequence database was constructed from the $50 \mathrm{bp}$ ends of each computationally predicted MspI-TaqI fragment in the $40-350$ bp size range. All Cs in each fragment were then converted to Ts; the converted reads were aligned to the converted reference. The number of mismatches in the induced alignment were counted between the unconverted read and reference, ignoring cases in which a $\mathrm{T}$ in the unconverted read matched to $\mathrm{a} \mathrm{C}$ in the unconverted reference. For a given read, the best alignment was kept if the second best alignment had 2 more mismatches; otherwise the read was discarded as nonunique. The methylation level of each sampled cytosine was estimated as the number of reads reporting a $\mathrm{C}$ divided by the total number of reads reporting a $\mathrm{C}$ or $\mathrm{T}$. Fisher's exact test or $t$-test was used for each $\mathrm{CpG}$ site that has at least 5 reads covered. Also, promoter, gene body, and CpG island annotations were added for each CpG. The software pipeline is implemented in Python.

2.5. Bisulfite Conversion and Multiplex Amplification. Samples were subjected to sodium bisulfite treatment using the EZ DNA Methylation-Direct ${ }^{\mathrm{TM}}$ Kit (Irvin, CA). Targeted amplification was done via Fluidigm 48, the 48 Access Array using Zymo Research's targeted sequencing service protocol. Sample loading, harvesting, and pooling were performed according to the manufacturer's protocol. Methylation profiling data from AA patients were compared with a normal candidate peripheral blood specimen as well as a normal colorectal tissue. A pairwise DNA methylation analysis was performed between colorectal neoplasia samples such as tubular adenoma, tubulovillous adenoma, and tumors versus normal tissue samples (blood and normal colon).

2.6. Barcoding/Adapterization PCR. Amplicon pools for each sample were diluted 1:100 and then amplified using barcoded adaptor-linkers received from Fluidigm according to the manufacturer's protocols (Fluidigm, San Francisco, CA). Reactions were cleaned up using the DNA Clean and Concentrator- 5 and the products were normalized by concentration and pooled. Sequencing, alignment, and data analysis libraries were denatured, diluted, and sequenced on the Illumina MiSeq according to the manufacturer's protocols (San Diego, CA). The sequencing run was a 150-base pairedend run. Sequence reads were aligned and analyzed as described above.

2.7. Pathway Analysis. Further analysis was performed using the Ingenuity Pathway Analysis (IPA) software. The IPA "Upstream Regulator Analysis" predicts upstream regulators by combining the directional methylation changes from our RRBS targeted methylation-sequencing and knowledge from prior experimental reports on causal effects between molecules such as TSG and oncogenes, compiled in the IPA Knowledge Base. Upstream Regulator Analysis calculates a $Z$-score based on the edge of dysregulation of all the downstream molecules and the uniformity of the existing evidence about the upstream-downstream relation, for every upstream regulator known to have a causal effect on at least 4 (activating and inhibiting Up/Down) dysregulated genes/ transcripts. $Z$-scores of $<0$ and $>0$, respectively, indicate a significant inhibition and activation state of the upstream regulator, regardless of the actual expression level of these molecules. See (http://pages.ingenuity.com/rs/ingenuity/images/0812\%20upstream_regulator_analysis_whitepaper.pdf.) The Network Generation Algorithm links molecules based on experimentally observed interactions and based on their interconnectedness. In general, the more interactions with other network members, the more central a molecule will be in a network.

\section{Results}

3.1. RRBS Global Methylation Analysis Revealed Candidate Genes. RRBS sequencing data from the 9 AA samples was obtained as described above and analyzed in pairwise fashion to determine differentially methylated $\mathrm{CpG}$ sites with relevance to colorectal neoplasia. This analysis showed increasing DNA methylation from normal to cancer with an average rate range from 0.09 (L3MBTL1) to 0.27 (PRDM14) (Table 2).

The RRBS analysis led to a list of genes that were differentially methylated in normal, tubular adenoma, tubulovillous adenoma, and colorectal neoplasia samples (Table 2). These genes (L3MBTL1, NKX6-2, PREX1, TRAF7, PRDM14, and NEFM) were methylated significantly in tumors when compared to normal tissue $(P<0.05)$.

The RRBS results for L3MBTL1 (NM_032107) showed 22 methylated $\mathrm{CpG}$ sites within the promoter region in 
TABLE 3: Number of methylated CpG sites at promoter of identified genes.

\begin{tabular}{lcccc}
\hline Genes & Normal & $\begin{array}{c}\text { Tubular } \\
\text { adenoma }\end{array}$ & $\begin{array}{c}\text { Tubulovillous } \\
\text { adenoma }\end{array}$ & Tumor \\
\hline L3MBTL1 & 0 & 0 & 0 & 22 \\
NKX6-2 & 0 & 0 & 0 & 37 \\
PREX1 & 0 & 0 & 6 & 27 \\
TRAF7 & 0 & 0 & 0 & 17 \\
PRDM14 & 0 & 0 & 0 & 28 \\
NEFM & 0 & 0 & 0 & 12 \\
\hline
\end{tabular}

tumor samples. None of the $\mathrm{CpG}$ sites were methylated in normal, tubular adenoma, and tubulovillous adenoma samples (Tables 2 and 3).

As for NKX6-2 (NM_177400), we identified 37 methylated $\mathrm{CpG}$ sites in promoter region in tumor samples, while none of these CpG sites were found to be methylated in normal, tubular adenoma, and tubulovillous adenoma samples (Tables 2 and 3 ).

For the PREX1 gene (NM_020820), we identified 27 methylated $\mathrm{CpG}$ sites in the promoter region in tumor samples and 6 methylated $\mathrm{CpG}$ sites in tubulovillous adenoma samples. None of these CpG sites were methylated in normal and tubular adenoma samples (Tables 2 and 3).

TRAF7 (NM_032271) displayed differential methylation for $17 \mathrm{CpG}$ sites in tumor samples, while none of these were methylated in normal, tubular adenoma, and tubulovillous adenoma samples (Tables 2 and 3).

The PRDM14 (NM_024504) gene displayed methylation in $28 \mathrm{CpG}$ sites in this gene's promoter region within tumor samples, while none of them was found to be methylated in normal, tubular adenoma, and tubulovillous adenoma samples (Tables 2 and 3).

The last identified marker in this study is NEFM (NM_005382) where 12 methylated CpG sites were identified within the gene's promotor region in tumor samples, while no CpG sites were found to be methylated in normal, tubular adenoma, and tubulovillous adenoma samples (Tables 2 and 3). Methylated CpG sites and their exact positions for the above genes are depicted in Figure 1.

3.2. Ingenuity Pathway Analysis. The hypermethylated markers were analyzed for their potential biological function(s) using the IPA software. L3MBTL1, NKX6-2, PREX1, TRAF7, PRDM14, and NEFM were mapped to Wnt/ $\beta$-catenin, P53, TGF- $\beta$, PI3k/AKT, VEGF, NF-k $\beta$, Hippo, P38 MAPK, and JAK/STAT3 signaling pathways (Table 4) (Figure 2).

3.3. Pathway Analysis of Differentially Methylated Genes. Running the upstream and downstream regulator analysis led to the identification of 6 crosstalk regulators (Table 4), namely, MMP3, GCG, HDAC1 and $A K T 1, D K G 4$, and SMAD2 that are affected by L3MBTL1, NKX6-2, PREX1, PRDM14, and NEFM, respectively. Both endogenous and exogenous molecules are included in this analysis. All significant regulators which are classified as transcription factors are displayed
TABle 4: (a) Significant regulators identified by IPA. (b) Genes' mapping to known molecular pathways.

(a)

\begin{tabular}{lc}
\hline Gene name & Crosstalk genes \\
\hline L3MBTL1 & MMP3 \\
NKX6-2 & GCG, CREB1 \\
PREX1 & HDAC1, AKT1 \\
TRAF7 & $I K B K B$ \\
PRDM14 & $D L G 4$ \\
NEFM & SMAD2 \\
\hline
\end{tabular}

(b)

\begin{tabular}{ll}
\hline Pathways & Differentially methylated genes \\
\hline Wnt/ $\beta$-catenin & ${ }^{*}$ L3MBTL1, NKX6-2, ${ }^{*}$ PREX1, ${ }^{*}$ TRAF7, and \\
& ${ }^{*}$ NEFM \\
P53 & L3MBTL1, PREX1, TRAF7, and NEFM \\
TGF- $\beta$ & L3MBTL1, PREX1, TRAF7, and NEFM \\
VEGF & L3MBTL1, PREX1, TRAF7, and NEFM \\
NF-k $\beta$ & PREX1, TRAF7, and NEFM \\
Hippo & PREX1, PRDM14, and NEFM \\
JAK/STAT3 & L3MBTL1, PREX1, TRAF7, and NEFM \\
P38 MAPK & L3MBTL1, NKX6-2, PREX1, TRAF7, \\
PI3K/AKT & PRDM14, and NEFM \\
\hline
\end{tabular}

${ }^{*}$ Common pathways.

in Table 4. All other regulator types can be found in Figure 2 as well. The significantly dysregulated molecules were sorted by their function or involvement in pathophysiological processes.

3.4. Network of Dysregulated Molecules Involved in IPAFunction. The following cellular functions have been potentially affected through the methylation of the identified 6 methylation targets (Figure 2).

Apoptosis Response. L3MBTL1, PREX1, TRAF7, PRDM14, and $N E F M$ are part of apoptosis response since they interact with P53, Hippo, and P38 MAPK pathways.

Proliferation Response. L3MBTL1, PREX1, TRAF7, and NEFM are part of proliferation response as they interact with TGF- $\beta$ and Hippo pathways.

Inflammatory Response. PREX1, TRAF7, and NEFM are part of inflammatory response because they interact with NF-k $\beta$ pathway.

\section{Discussion}

Many approaches have been used to detect the status of DNA methylation in human tissues. Most of these methods relied on the preknowledge of the analyzed methylation targets such as MLH1, p16, and other known genes in colon neoplastic transformation. Herein, we used Reduced 


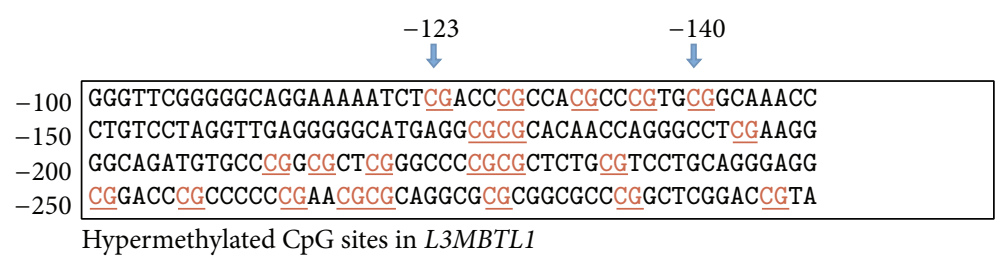

(a)

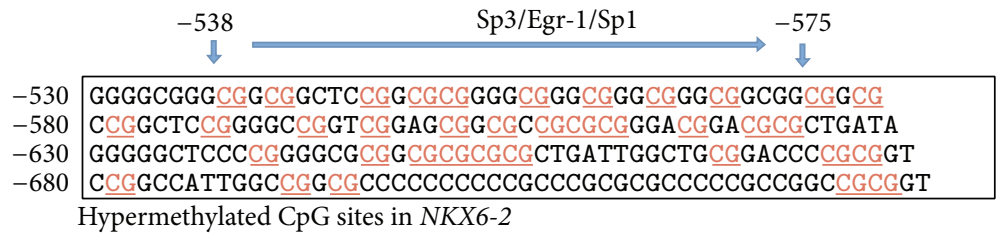

(b)

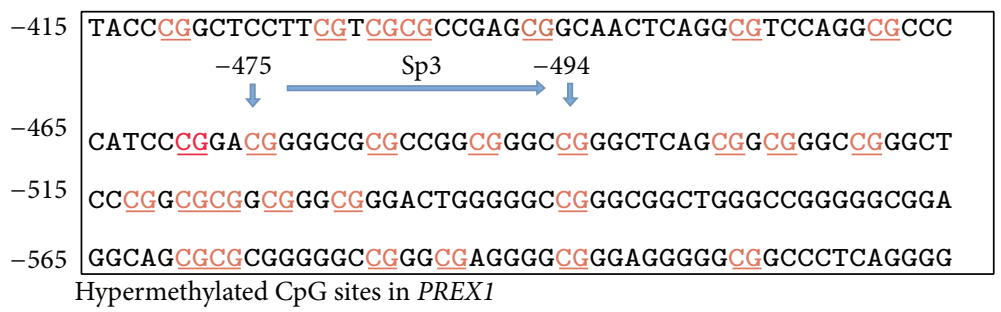

(c)

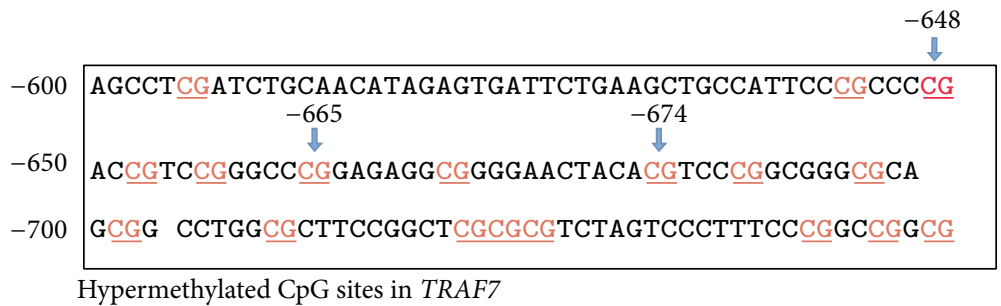

(d)

\begin{tabular}{|c|c|}
\hline-560 & $\begin{array}{c}\text { AGCCATCGGCCCACGCAGATCCCCGCGTCGTCTTCTCCCCGCCCACCGCT } \\
-620 \stackrel{\mathrm{NF}-\mathrm{Y}}{\longrightarrow}-627\end{array}$ \\
\hline & GGCCGACTCTCGGGCGACGGGCGGGAGGGGGCGGCCCTCCCGGGCGGCG \\
\hline
\end{tabular}

Hypermethylated CpG sites in PRDM14

(e)

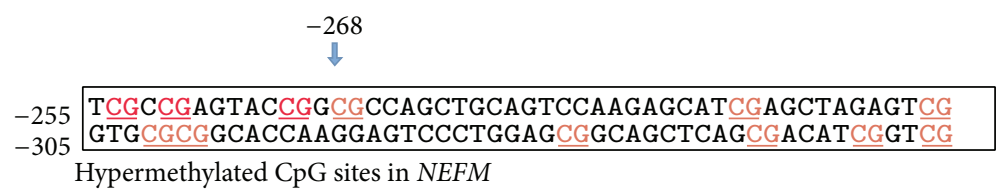

(f)

FIGURE 1: DNA methylation profile of RRBS analyzed hypermethylated genes in the tumor samples of African American patients: L3MBTL1 (a), NKX6-2 (b), PREX1 (c), TRAF7 (d), PRDM14 (e), and NEFM (f).

Representation Bisulfite Sequencing (RRBS) to establish differential DNA methylation targets in the path to colorectal cancer. RRBS has single-base resolution and detects CpG sites in gene bodies as well as intergenic regions, CpG islands and nonisland sequences, through highly sensitive sequencing of pooled sodium bisulfite modified CpG sequences [20]. The implementation of this technique within our study led to the identification of several potential markers that were selected based on the analysis of different combinations of samples (normal versus adenoma, adenoma versus cancer, and normal versus cancer). Here we identified specific $\mathrm{CpG}$ sites in L3MBTL1, NKX6-2, PREX1, TRAF7, PRDM14, and $N E F M$ as primarily methylated in cancer specimens but not in other colonic lesions; these genes were also found to be implicated in many important pathways relevant to neoplastic transformation. 


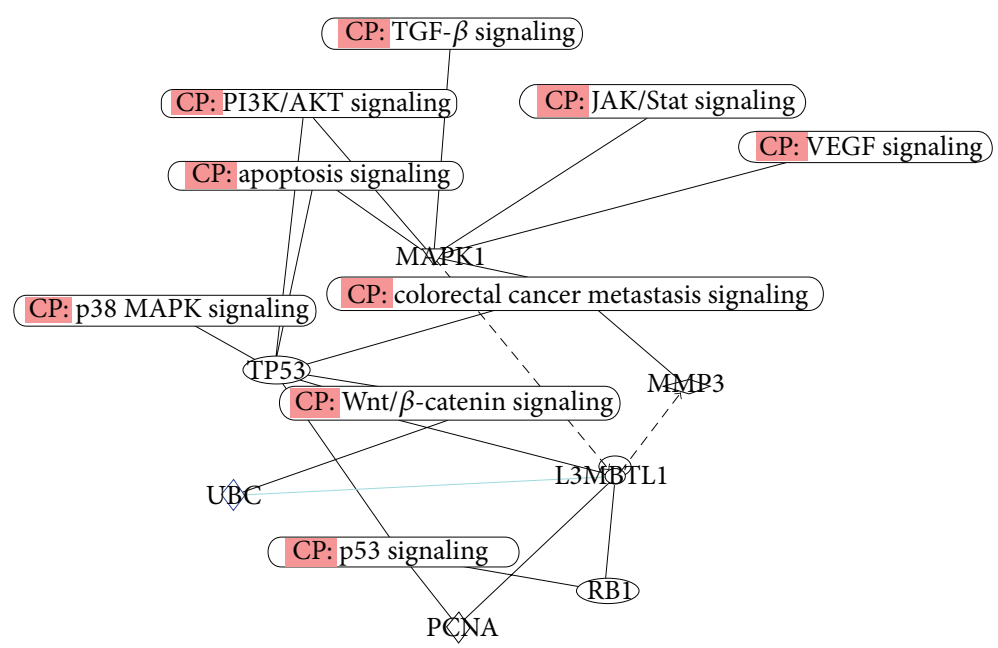

(a) L3MBTL1

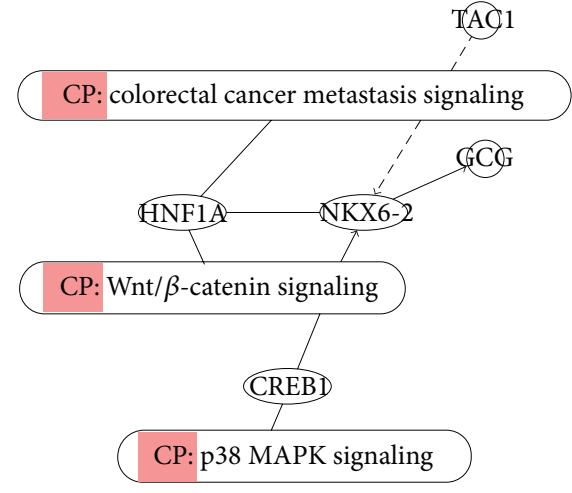

๑) 2000-2015 QIAGEN. All rights reserved.

(b) NKX6-2

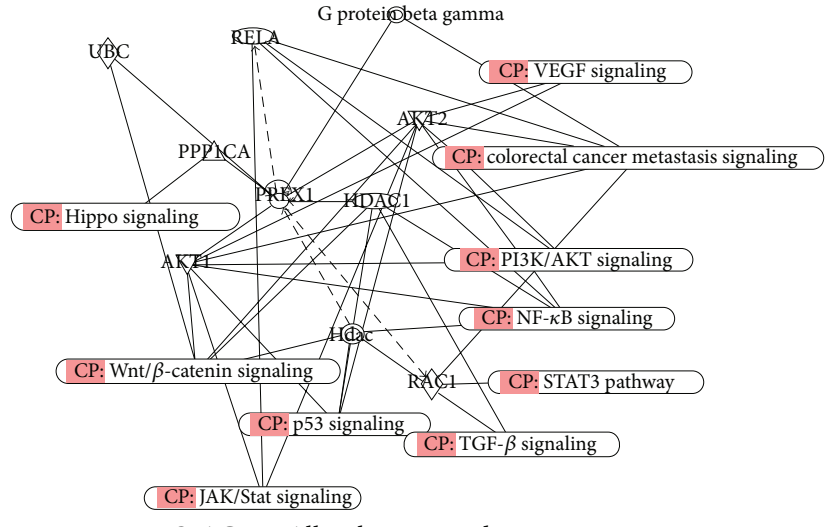

(c) 2000-2015 QIAGEN. All rights reserved.

(c) PREX1

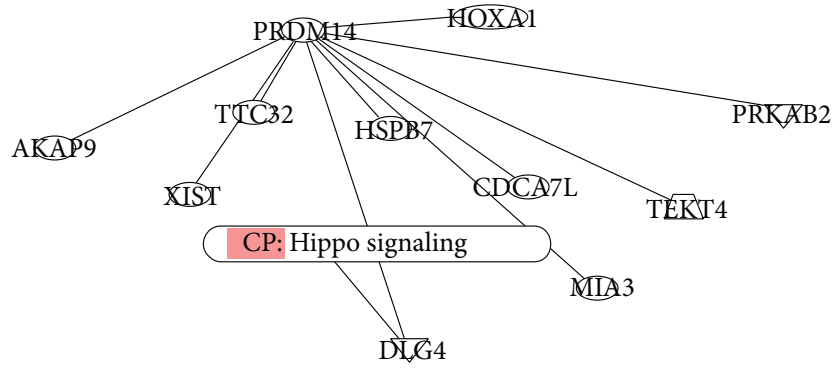

(e) PRDM14

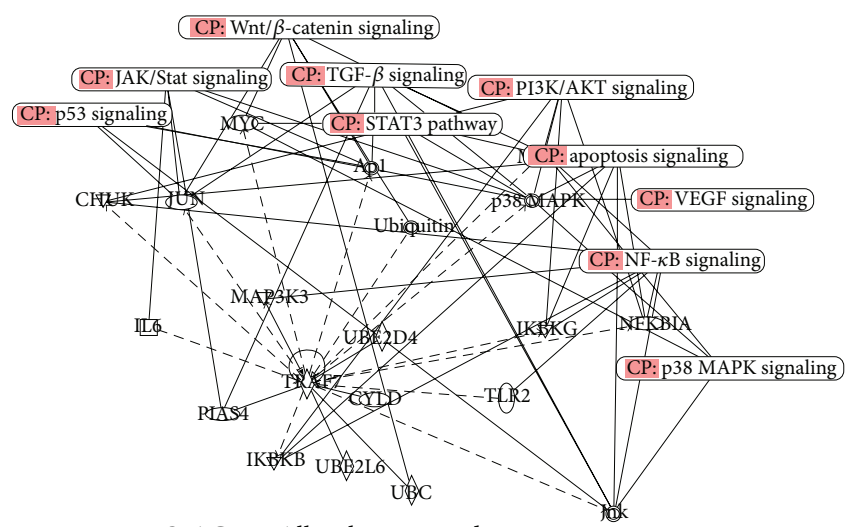

(c) 2000-2015 QIAGEN. All rights reserved.

(d) TRAF7

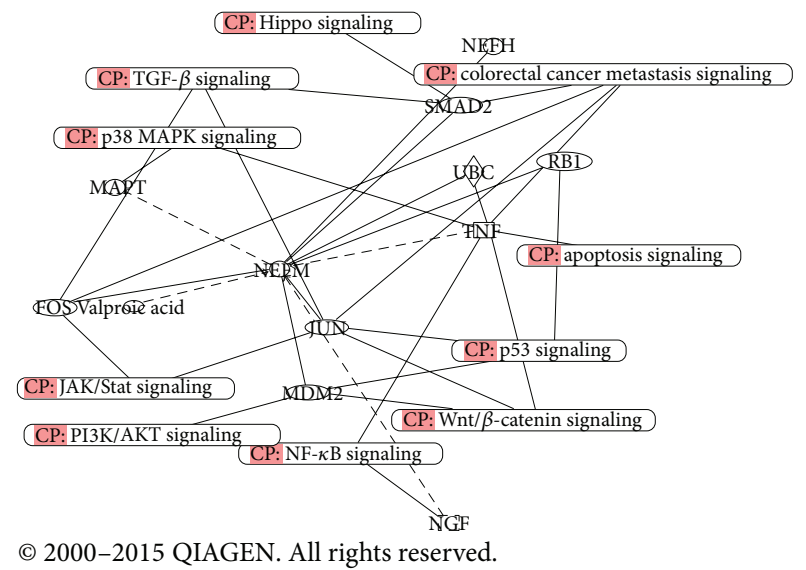

(f) NEFM

FIgURE 2: Ingenuity Pathways Analysis of the identified methylated targets. L3MBTL1 (a), NKX6-2 (b), PREX1 (c), TRAF7 (d), PRDM14 (e), and NEFM (f). CP: Conical Pathway.

L3MBTL1 gene located on chromosome 20q12 is a tumor suppressor gene. It is known to act as chromatin transcription repressor, which is activated mainly in germline stem cells [21]. This gene, which belongs to the polycomb group (PcG) proteins, binds to several methylated lysines in $\mathrm{H} 1 \mathrm{~b}, \mathrm{H} 3$, and
H4, blocking DNA sequences from access to transcription [21]. Zeng et al. found that high levels of L3MBTL1 methylation were related to slightly elevated risk of breast cancer death, and hypermethylation of L3MBTL1 in breast cancer patients can be reduced through physical exercise which 
positively associated with overall survival [21]. IPA analysis mapped this gene within a network of Wnt/ $\beta$-catenin, P53, TGF- $\beta$, VEGF, P38 MAPK, and JAK/STAT3. In our study, this marker's methylation was noted in cancer but not adenoma samples which is in line with the type of pathways it is involved with through IPA analysis. To our knowledge, this is the first implication of this gene in colorectal cancer. If found in any preneoplastic lesions, this gene's methylation should point to highly carcinogenic lesions that need to be addressed and monitored aggressively due to its implication with major cancer pathways, including VEGF that points to metastasis implications as well as NKX6-2 gene located on chromosome 10 , known as a prognostic marker. Previous studies showed that NKX6-2 is hypermethylated in bladder tumors and hypomethylated in normal leukocytes [22]. Another study demonstrated that DNA hypermethylation of NKX6-2 is a hallmark of CIMP at very early stages of renal carcinogenesis [23]. NKX6-2 was also identified as a biomarker in stages II and III lung adenocarcinoma, where it was hypomethylated in stage II and hypermethylated in stage III [24]. The CpG sites from -538 to -575 upstream of NKX6-2 are within the binding sites of SP1, SP3, and Egr-1 (Figure 1(b)) [25]. SP1 has been reported to serve as a transcriptional activator while SP3 acts as both an activator and a repressor, depending on cell and tissue type [25]. DNA methylation of promoter regions is associated with transcriptional silencing, either by preventing the binding of specific transcription factors or through the recruitment of methyl CpG binding proteins [25]. Egr-1 and SP1 are known to act as positive regulators of NKX6-2; this might elucidate the effect of these transcription factors on NKX6-2 gene [26]. IPA mapped this gene within the Wnt/ $\beta$ catenin and P38 MAPK signaling pathways. We here report the first implication of this gene's methylation in colorectal carcinogenesis. The impact of the hypermethylated NKX6-2 gene has been reported in bladder cancer, renal cancer, and lung adenocarcinoma, and this is consistent with our results in colorectal cancer specimens.

TRAF7 is an E3 ubiquitin ligase for several proteins which is located in chromosome 16P. A study showed that downregulation of TRAF7 is correlated with poor prognosis in breast cancer development [27]. IPA mapped this gene within a network of Wnt/ $\beta$-catenin, P53, TGF- $\beta$, PI3K/AKT, VEGF, NF-k $\beta$, Hippo, P38 MAPK, and JAK/STAT3 signaling pathways. Here, we showed that hypermethylation of TRAF7 is associated with cancer, but not preneoplastic specimens. To our knowledge this is the first time this gene is cited as a distinctive gene in CRC patients. The impact of the hypermethylated TRAF7 gene has been reported in breast cancer and it is consistent with our results that this gene plays an important role in colorectal carcinogenesis.

PRDM14 contains a PR domain that is likely a derivative of SET domain, which is involved in the methylation of lysine residues on the histone tail, and affects chromatin structure and gene expression [28]. A retrospective study showed that PRDM14 is frequently overexpressed in breast cancers and that its overexpression is often associated with gene amplification [28]. We here report that this gene might also be a target of methylation in colorectal cancer. The present in silica study also suggests that DNA methylation of a CpG site located -620 to $-627 \mathrm{bp}$ upstream from the transcription start site in the promoter of PRDM14 may affect the expression of this gene [29]. CpG site -620 to -627 is located in the binding site to which NFY can bind (Figure 1(e)) [30]. Nuclear transcription factor Y (NFY) is a protein forming a highly conserved transcription factor in the promoter regions of a variety of genes. Previous studies suggested that NFY plays an important role in the regulation of genes that are expressed in various types of cancers and its involvement in tumor metastasis and breast cancer progression has been noted [30]. Moreover, NFY appears to regulate the expression of cell cycle and cell death related genes, suggesting its contribution to tumor cell proliferation [30]. IPA mapped this gene within a network of Hippo and P38 MAPK signaling pathways. The effect of the aberrant methylation of PRDM14 gene has been reported in breast cancer and it is consistent with our results that hypermethylation of this gene plays an important role in many cancers.

$N E F M$ is a gene located in chromosome $8 \mathrm{p}$ and is hypermethylated in renal cell carcinoma [31]. IPA mapped this gene within a network of Wnt/ $\beta$-catenin, P53, TGF- $\beta$, PI3K/AKT, NF-k $\beta$, P38 MAPK, and JAK/STAT3 signaling pathways. To our knowledge this is the first time that NEFM is reported as a methylation target in colorectal cancer. The impact of the hypermethylated NEFM gene has been reported in renal cancer, and it is consistent with our results that this gene plays an important role in colorectal carcinogenesis. We are aware of the limitations of this study, such as the sample size, which is primarily due to the high costs associated with the RRBS methodology. As the technology becomes more accessible and cost effective, we plan larger size studies to validate the reported findings.

In conclusion, we here report six new distinct $\mathrm{CpG}$ methylation targets (L3MBTL1, NKX6-2, PREX1, TRAF7, PRDM14, and NEFM) in African Americans with CRC. These markers were found to be involved in major carcinogenic pathways and may be of a potential interest as CRC marker in African Americans.

\section{Competing Interests}

The authors declare that they have no competing interests.

\section{Acknowledgments}

This project was supported (in part) by the National Institute on Minority Health and Health Disparities of the National Institutes of Health under Award no. G12MD007597. This project was also funded in part with Federal Funds (Grant no. UL1TR000101, previously UL1RR031975) from the National Center for Advancing Translational Sciences (NCATS), National Institutes of Health (NIH), through the Clinical and Translational Science Awards Program.

\section{References}

[1] K. Kumar, H. Brim, F. Giardiello et al., "Distinct BRAF (V600E) and KRAS mutations in high microsatellite instability 
sporadic colorectal cancer in African Americans," Clinical Cancer Research, vol. 15, no. 4, pp. 1155-1161, 2009.

[2] S. Al-Sohaily, A. Biankin, R. Leong, M. Kohonen-Corish, and J. Warusavitarne, "Molecular pathways in colorectal cancer," Journal of Gastroenterology and Hepatology, vol. 27, no. 9, pp. 1423-1431, 2012.

[3] H. Ashktorab, M. Daremipouran, A. Goel et al., "DNA methylome profiling identifies novel methylated genes in African American patients with colorectal neoplasia," Epigenetics, vol. 9, no. 4, pp. 503-512, 2014.

[4] Surveillance, Epidemiology, and End Results Program 2015, http://seer.cancer.gov/.

[5] M. Esteller, P. G. Corn, S. B. Baylin, and J. G. Herman, "A gene hypermethylation profile of human cancer," Cancer Research, vol. 61, no. 8, pp. 3225-3229, 2001.

[6] E. N. Mojarad, P. J. K. Kuppen, H. A. Aghdaei, and M. R. Zali, "The CpG island methylator phenotype (CIMP) in colorectal cancer," Gastroenterology and Hepatology from Bed to Bench, vol. 6, no. 3, pp. 120-128, 2013.

[7] S. D. Markowitz and M. M. Bertagnolli, "Molecular basis of colorectal cancer," The New England Journal of Medicine, vol. 361, no. 25, pp. 2404-2460, 2009.

[8] H. Ashktorab, H. Rahi, D. Wansley et al., "Toward a comprehensive and systematic methylome signature in colorectal cancers," Epigenetics, vol. 8, no. 8, pp. 807-815, 2013.

[9] Y. Zhang, Q. Li, and H. Chen, "DNA methylation and histone modifications of Wnt genes by genistein during colon cancer development," Carcinogenesis, vol. 34, no. 8, pp. 1756-1763, 2013.

[10] L. Shen, H. Kantarjian, Y. Guo et al., "DNA methylation predicts survival and response to therapy in patients with myelodysplastic syndromes," Journal of Clinical Oncology, vol. 28, no. 4, pp. 605-613, 2010.

[11] Y. Suehiro, C. W. Wong, L. R. Chirieac et al., "Epigenetic-genetic interactions in the APC/WNT, RAS/RAF, and P53 pathways in colorectal carcinoma," Clinical Cancer Research, vol. 14, no. 9, pp. 2560-2569, 2008.

[12] P. Mokarram, K. Kumar, H. Brim et al., "Distinct high-profile methylated genes in colorectal cancer," PLOS ONE, vol. 4, no. 9, article e7012, 2009.

[13] D. J. Weisenberger, K. D. Siegmund, M. Campan et al., "CpG island methylator phenotype underlies sporadic microsatellite instability and is tightly associated with BRAF mutation in colorectal cancer," Nature Genetics, vol. 38, no. 7, pp. 787-793, 2006.

[14] D. Colussi, G. Brandi, F. Bazzoli, and L. Ricciardiello, "Molecular pathways involved in colorectal cancer: implications for disease behavior and prevention," InternationaL Journal of Molecular Sciences, vol. 14, no. 8, pp. 16365-16385, 2013.

[15] K. E. Schuebel, W. Chen, L. Cope et al., "Comparing the DNA hypermethylome with gene mutations in human colorectal cancer," PLoS Genetics, vol. 3, no. 9, pp. 1709-1723, 2007.

[16] Y. Li, J. Deuring, M. P. Peppelenbosch, E. J. Kuipers, C. de Haar, and C. J. van der Woude, "IL-6-induced DNMT1 activity mediates $\mathrm{SOCS}_{3}$ promoter hypermethylation in ulcerative colitis-related colorectal cancer," Carcinogenesis, vol. 33, no. 10, pp. 1889-1896, 2012.

[17] M. P. A. Ebert, M. Tänzer, B. Balluff et al., “TFAP2E-DKK4 and chemoresistance in colorectal cancer," The New England Journal of Medicine, vol. 366, no. 1, pp. 44-53, 2012.

[18] Z.-M. Zhang, Y. Wang, R. Huang et al., “TFAP2E hypermethylation was associated with survival advantage in patients with colorectal cancer," Journal of Cancer Research and Clinical Oncology, vol. 140, no. 12, pp. 2119-2127, 2014.

[19] E. Miotto, S. Sabbioni, A. Veronese et al., "Frequent aberrant methylation of the $\mathrm{CDH} 4$ gene promoter in human colorectal and gastric cancer," Cancer Research, vol. 64, no. 22, pp. 8156$8159,2004$.

[20] K. Tsumagari, C. Baribault, J. Terragni et al., "Early de novo DNA methylation and prolonged demethylation in the muscle lineage," Epigenetics, vol. 8, no. 3, pp. 317-332, 2013.

[21] H. Zeng, M. L. Irwin, L. Lu et al., "Physical activity and breast cancer survival: an epigenetic link through reduced methylation of a tumor suppressor gene L3MBTL1," Breast Cancer Research and Treatment, vol. 133, no. 1, pp. 127-135, 2012.

[22] W. Chung, J. Bondaruk, J. Jelinek et al., "Detection of bladder cancer using novel DNA methylation biomarkers in urine sediments," Cancer Epidemiology Biomarkers and Prevention, vol. 20, no. 7, pp. 1483-1491, 2011.

[23] E. Arai, S. Chiku, T. Mori et al., "Single-CpG-resolution methylome analysis identifies clinicopathologically aggressive CpG island methylator phenotype clear cell renal cell carcinomas," Carcinogenesis, vol. 33, no. 8, pp. 1487-1493, 2012.

[24] M. P. Pradhan, A. Desai, and M. J. Palakal, "Systems biology approach to stage-wise characterization of epigenetic genes in lung adenocarcinoma," BMC Systems Biology, vol. 7, article 141, 2013.

[25] E. Hall, T. Dayeh, C. L. Kirkpatrick, C. B. Wollheim, M. Dekker Nitert, and C. Ling, "DNA methylation of the glucagon-like peptide 1 receptor (GLP1R) in human pancreatic islets," $B M C$ Medical Genetics, vol. 14, no. 1, article 76, 2013.

[26] M. Liu, X. Wang, Y. Peng, S. Shen, and G. Li, "Egr-1 regulates the transcription of NGX6 gene through a Sp1/Egr-1 overlapping site in the promoter," BMC Molecular Biology, vol. 15, no. 1, article 14, 2014.

[27] L. Wang, L. Wang, S. Zhang et al., "Downregulation of ubiquitin E3 ligase TNF receptor-associated factor 7 leads to stabilization of p53 in breast cancer," Oncology Reports, vol. 29, no. 1, pp. 283287, 2013.

[28] N. Nishikawa, M. Toyota, H. Suzuki et al., "Gene amplification and overexpression of PRDM14 in breast cancers," Cancer Research, vol. 67, no. 20, pp. 9649-9657, 2007.

[29] M. Risio, G. Reato, P. F. Di Celle, M. Fizzotti, F. P. Rossini, and R. Foà, "Microsatellite instability is associated with the histological features of the tumor in nonfamilial colorectal cancer," Cancer Research, vol. 56, no. 23, pp. 5470-5474, 1996.

[30] MAPPER, Multi-Genome Analysis of Positions and Patterns of Elements of Regulation, 2015.

[31] C. J. Ricketts, M. R. Morris, D. Gentle et al., "Methylation profiling and evaluation of demethylating therapy in renal cell carcinoma," Clinical Epigenetics, vol. 5, no. 1, article 16, 2013. 


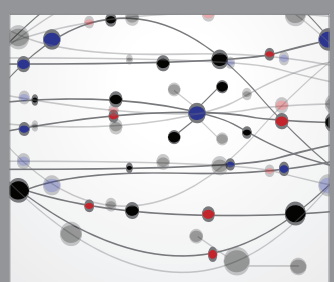

The Scientific World Journal
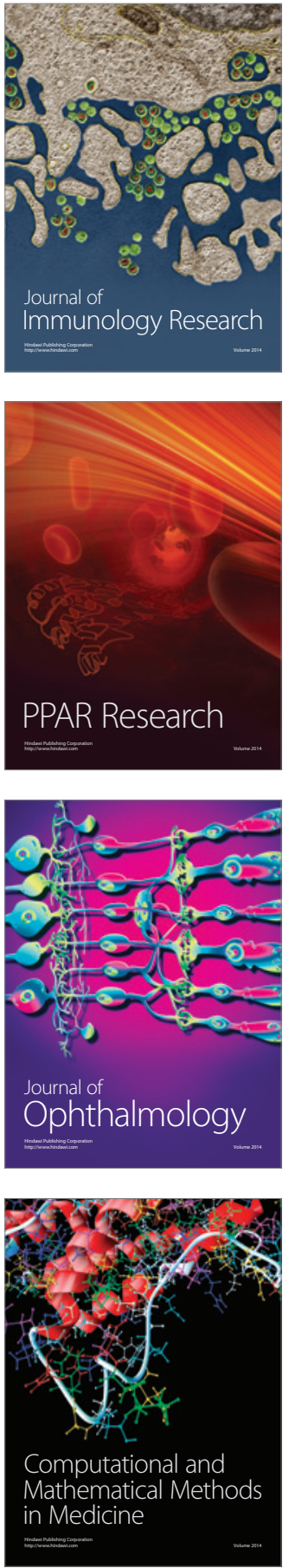

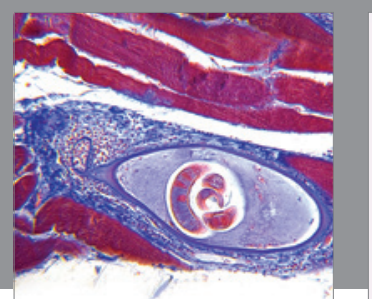

Gastroenterology Research and Practice

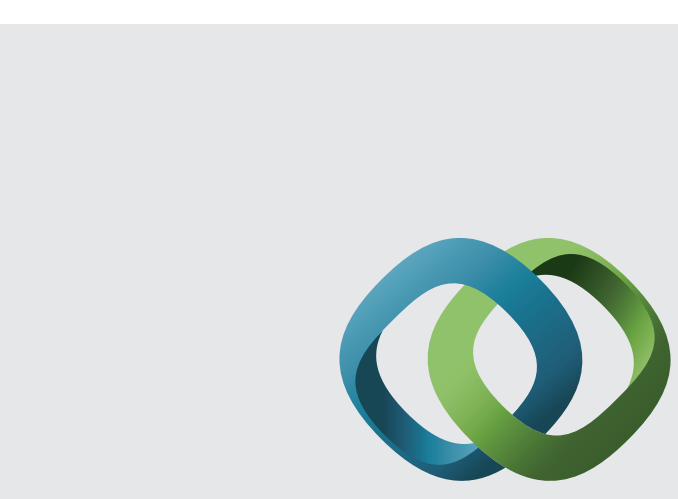

\section{Hindawi}

Submit your manuscripts at

http://www.hindawi.com
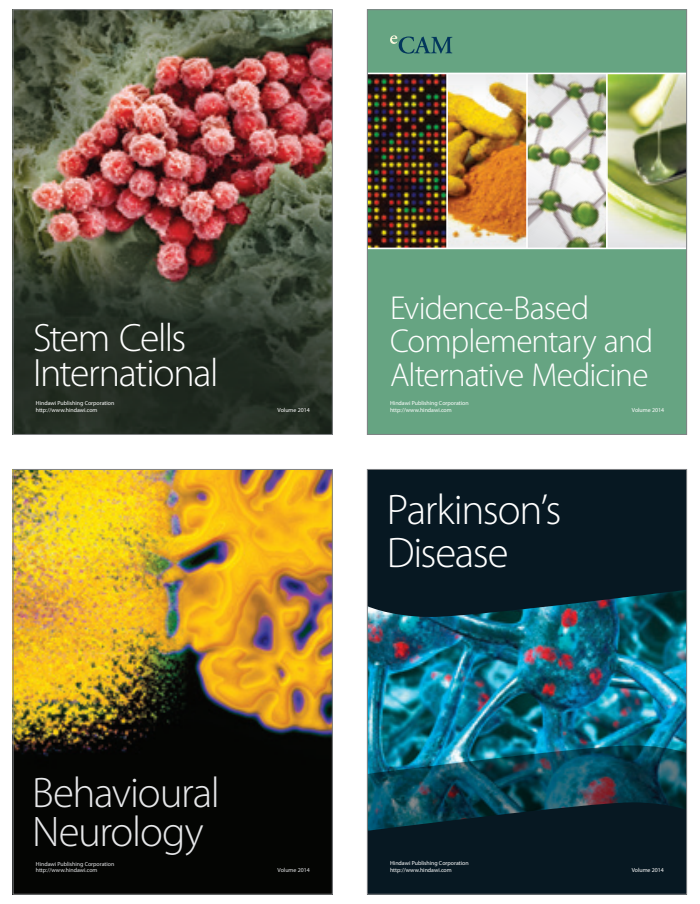
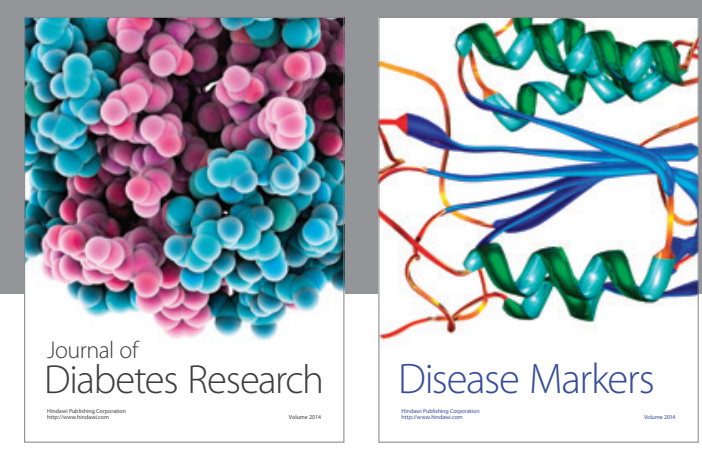

Disease Markers
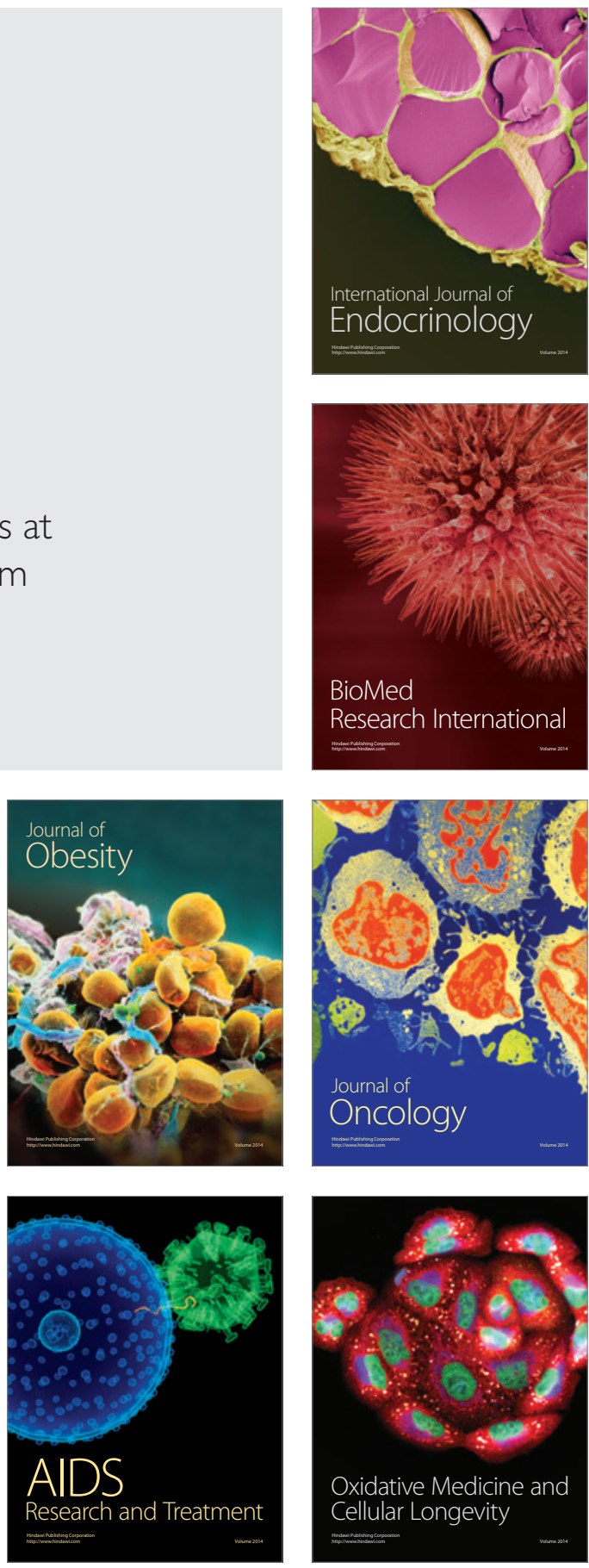